\title{
Improving the management of spontaneous bacterial peritonitis in cirrhotic patients: assessment of an intervention in trainee doctors 垱”
}

\author{
Authors: Timothy M Rawson, ${ }^{A}$ Sonia Bouri, ${ }^{B}$ Christopher Allen, ${ }^{C}$ João Ferreira-Martins, ${ }^{D}$ Abulkani Yusuf, ${ }^{E}$ \\ Nina Stafford, ${ }^{F}$ Maxton Pitcher $^{G}$ and Meron Jacyna ${ }^{H}$
}

Spontaneous bacterial peritonitis (SBP) in cirrhotic patients is a serious complication associated with a high mortality rate. A baseline audit of the acute medical take (AMT) at Northwick Park suggested a lack of awareness regarding management. A questionnaire based on contemporary SBP guidelines was circulated to all trainee doctors (FY1 to SpR). Ascitic fluid testing requests were analysed over a six-month period. The electronic requesting system was updated to include prompts and direct links to Trust SBP guidelines, and a one-hour lecture to all members of the AMT, supported by an educational booklet on SBP, was performed. Re-audit was carried out six months postintervention, the AMT completed a second questionnaire and ascitic fluid testing requests were re-audited. In comparable pre- and post-intervention AMT cohorts, a clinical and educational intervention led to a significant improvement in understanding of when to investigate $(p \leq 0.001)$, samples $(p=0.002)$ and containers $(p \leq 0.001)$ required, urgency of obtaining results $(p \leq 0.001)$, and initiation of treatment for suspected SBP ( $p=0.007)$. Significantly more ascitic samples were sent, with specific suspicion of SBP more readily documented, crucial to expediting laboratory processing. Targeted education and production of a clinical algorithm has significantly improved the management of patients with SBP.

KEYWORDS: Spontaneous bacterial peritonitis, infection management, diagnostics, guidelines, educational intervention, clinical audit

Authors: Aacademic foundation year trainee, Northwick Park Hospital, Harrow, UK; ${ }^{B}$ Core medical trainee, Northwick Park Hospital, Harrow, UK; ' Core medical trainee, Northwick Park Hospital, Harrow, UK; Dfoundation year trainee, Northwick Park Hospital, Harrow, UK; ${ }^{E}$ specialist registrar in gastroenterology, Northwick Park Hospital, Harrow, UK; F Fpecialist registrar in gastroenterology, Northwick Park Hospital, Harrow, UK; ${ }^{G}$ Consultant gastroenterologist, Northwick Park Hospital, Harrow, UK; ${ }^{H}$ Consultant gastroenterologist, Northwick Park Hospital, Harrow, UK

\section{Introduction}

One of the most serious complications of cirrhosis is the development of spontaneous bacterial peritonitis (SBP). Untreated, it carries a mortality of up to $90 \%,{ }^{1}$ which falls to $20 \%$ with prompt recognition and early treatment. ${ }^{1,2}$ It is a common complication of cirrhosis - the prevalence in cirrhotic in-patients varies from $10-30 \%{ }^{1,2}-$ and the clinical presentation is diverse, with abdominal pain, fever, upper gastrointestinal haemorrhage or an asymptomatic presentation all commonly observed. ${ }^{1,2}$ Given this variation and high mortality, a high index of clinical suspicion for possible SBP, accompanied by a rapid diagnostic algorithm is required. Moreover, with cirrhosis-related admissions increasing in the $\mathrm{UK},{ }^{4,5}$ a greater responsibility falls on the clinician to identify risk factors and initiate swift and appropriate management. ${ }^{4}$

We aimed to investigate the knowledge of SBP, in trainee doctors involved in the acute medical take (AMT) at Northwick Park Hospital, London, UK.

\section{Method}

Using the European Association for the Study of the Liver (EASL) and British Society of Gastroenterology (BSG) clinical practice guidelines for SBP, ${ }^{1,2}$ we developed a six-point questionnaire (Box 1). This tested trainee doctors' knowledge of when to suspect SBP, how to investigate it (including correct samples to send to the laboratory), the urgency of results, appropriate treatment and current guidelines available for them to use. This was circulated to all trainee doctors, from foundation year 1 (FY1) to specialist registrar (SpR), involved in the AMT at Northwick Park Hospital in January 2014.

We reviewed all ascitic samples received by the microbiology laboratory between May 2013 and January 2014 and selected all patients with cirrhosis. White cell count (WCC), differential count (polymorphonuclearcyte (PMN) and lymphocyte percentages) and culture results were obtained using the electronic results system. Time between admission and diagnostic paracentesis was noted. Time between samples being recorded as received by the laboratory and being electronically reported was also documented. Documentation of clinical suspicion on sample request labels was recorded. 
Box 1. Questionnaire provided to trainees involved in the initial management of cirrhotic inpatients with ascites in the acute medical take.

1. In which patients should you routinely perform ascitic paracentesis to exclude spontaneous bacterial peritonitis (SBP)?

$\square$ Any cirrhotic patient with ascites on admission to hospital

$\square$ Only if cirrhotic with ascites AND clinical suspicion (fever, pains, sepsis)

2. What tests (including specific containers) would you send off for suspected SBP?

\begin{tabular}{|l|l|}
\hline Tests required & Sample container required \\
\hline & \\
\hline & \\
\hline & \\
\hline
\end{tabular}

3. How do you do you diagnose SBP?

4. When would you start treatment if you suspected SBP?

$\square$ As soon as possible before diagnostic paracentesis

$\square$ After diagnostic paracentesis

5. How quickly do you require a WCC result from diagnostic paracentesis?

$\square$ Within 24 hours

$\square$ Routine

$\square$ Special request from laboratory

6. What guidance are you aware of regarding diagnostic paracentesis and SBP?

The results were reviewed by a panel of doctors, including trainees (FY1 to SpR) and consultants. A number of initiatives, aimed at improving trainee understanding and minimising delays and diagnosis of SBP were implemented. In July 2014 (six months post-intervention), trainees involved in the AMT once again completed the six-point questionnaire and a review of electronic records of all ascitic samples from cirrhotic inpatients taken between February and July 2014 was performed. Results were compared to those preintervention. Where appropriate, statistical analysis was performed using Chi-squared or Mann-Whitney U statistical analysis.

\section{Interventions performed}

Following assessment of trainee doctors' knowledge and reviews of ascitic samples sent to the laboratory, a number of interventions were implemented.

\section{Clinical}

A local guideline based on current EASL and BSG guidance was developed. ${ }^{1,2}$ This included a diagnostic and management algorithm for SBP (Fig 1), as well as post-diagnosis management recommendations (not included). These included local microbiology guidelines on management of SBP, which were unchanged between interventions. This was uploaded onto our Trust intranet for ease of access. Second, the electronic requesting system was updated to include a prompt with every ascitic fluid analysis request. This prompt asks whether the patient 'is known to have cirrhosis'. If the trainee then answers 'yes' the system displays an alert reminding them that they should have a high suspicion of SBP and urgently seek out the results of the investigation by contacting the laboratory. It also provides the trainee with a direct link to the Trust guidelines on diagnosis and management of SBP and provides a written investigation order form detailing the specific samples to be obtained.

\section{Educational}

A senior SpR in gastroenterology delivered a one-hour lecture to all members of the AMT on SBP. This was supported by the creation of a 20-page, educational booklet on SBP which was provided to the trainee doctors at the session. It is designed to be worked through in the trainees' own time, aiming to draw on previous knowledge of SBP learnt during medical training and refresh the key points of the topic to aid identification and management of this serious complication. On completion of this booklet the trainee should be able to (learning outcomes):

1. Define SBP and its pathogenesis

2. Understand the epidemiology and clinical presentation of SBP

3. Understand the correct pathway for investigation of SBP

a. correct investigations to perform

b. correct laboratory samples required to send

4. Describe the correct management of patients with suspected SBP

5. Be aware of guidance and current evidence surrounding this topic

\section{Results}

Pre-intervention

In total, 34 trainee doctors (17 FY1s, 15 senior house officers (SHOs) and $2 \mathrm{SpRs}$ ) from the AMT completed our questionnaire on SBP in cirrhotic inpatients. 15 (44\%) had performed between 1 and 5 diagnostic paracenteses previously, $6(20 \%)$ had performed $>5$ procedures, while 13 trainees $(38 \%)$ had no experience. Only 4 (12\%) trainees correctly answered that all known cirrhotic inpatients should have a paracentesis performed on admission to hospital; 16 (47\%) knew which investigations on ascitic fluid samples were required, although only $2(6 \%)$ where able to recall the correct sample containers for the tests; 12 trainees (35\%) knew the correct definition for diagnosis of SBP and when to initiate treatment; 13 (38\%) trainees understood that white cell counts should be requested as urgent from the laboratory and only 5 (15\%) could correctly name a source of guidance on the diagnosis and management of SBP. Table 1 provides a comparison of trainees knowledge pre- and post-interventions designed to increase knowledge and 
垱”滔 Timothy M Rawson, Sonia Bouri and Christopher Allen et al

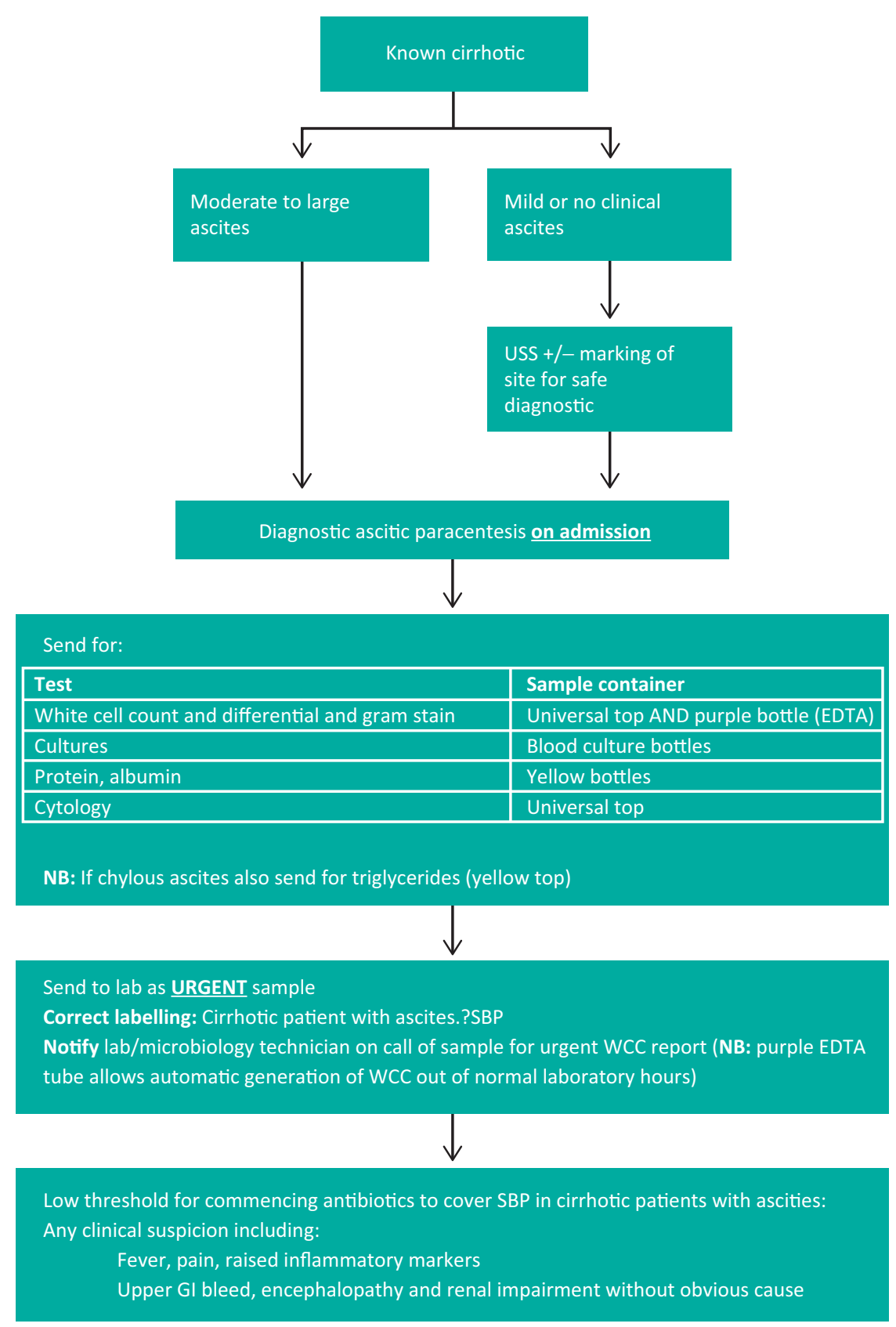

Fig 1. Guideline introduced to the Trust: ascites investigation pathway for cirrhotic inpatients. EDTA = ethylenediaminetetraacetic acid; $\mathrm{GI}=$ gastrointestinal; $\mathrm{SBP}=$ spontaneous bacterial peritonitis; USS = ultrasound scan; $\mathrm{WCC}=$ white cell count. understanding surrounding the management of ascites and SBP in cirrhotic inpatients.

\section{Post-intervention}

26 trainee doctors (11 FY1, 14 SHO and 1 SpR) on the AMT completed our questionnaire. The pre- and post-intervention cohorts did not differ in experience, with respect to trainee grade or number of paracenteses performed $(\mathrm{p}=0.83$ and $\mathrm{p}=0.31$, respectively). However, there was a significant improvement in understanding of when to perform ascitic paracentesis in cirrhotic patients, with 58\% (15) answering correctly post-intervention compared for $11 \%$ (4) pre-intervention $(\mathrm{p} \leq 0.001)$. Knowledge of the correct samples required improved from $47 \%$ (16) to $85 \%$ (22) and correct containers required improved from $6 \%$ (2) to $54 \%$ (14) postintervention $(\mathrm{p}=0.002$ and $\mathrm{p} \leq 0.001$, respectively). Awareness of the urgency of obtaining WCC results rose from $38 \%$ (13) to $73 \%(19)(\mathrm{p} \leq 0.001)$ and trainees were also more aware of when to initiate treatment for suspected SBP (62\% from $35 \%, \mathrm{p}=0.007)$. Knowledge of availability of where to locate appropriate guidance on management of SBP also increased from $15 \%$ (5) to $81 \%$ (21) following intervention ( $p \leq 0.001$ ). There was no difference in antibiotic selection between interventions, with appropriate choices made based on local microbiology guidelines pre- and post-intervention. 
Table 1. Comparison of trainees understanding of the management of cirrhotic inpatients with cirrhosis and spontaneous bacterial peritonitis before and after intervention.

\begin{tabular}{|c|c|c|c|c|}
\hline Question & Answer & $\begin{array}{l}\text { Pre-intervention, } \\
n(\%)(n=34)\end{array}$ & $\begin{array}{l}\text { Post-intervention, } \\
n(\%)(n=26)\end{array}$ & $\mathrm{p}$ value \\
\hline \multirow[t]{3}{*}{ Grade } & FY1 & $17(50)$ & $11(42)$ & \\
\hline & $\mathrm{SHO}$ & $15(44)$ & $14(54)$ & \\
\hline & $\mathrm{SpR}$ & $2(6)$ & $1(4)$ & 0.830 \\
\hline \multirow[t]{3}{*}{ Number of taps performed } & 0 & $13(38)$ & $7(28)$ & \\
\hline & $0-5$ & $15(44)$ & $10(38)$ & \\
\hline & $>5$ & $6(18)$ & $9(35)$ & 0.310 \\
\hline Understand of when to perform paracentesis & Correct & $4(11)$ & $15(58)$ & $<0.001$ \\
\hline \multirow[t]{2}{*}{ Correct tests to order } & Correct & $16(47)$ & $22(85)$ & 0.002 \\
\hline & Partially correct & $14(41)$ & $4(15)$ & \\
\hline \multirow[t]{2}{*}{ Correct sample containers } & Correct & $2(6)$ & $14(54)$ & $<0.001$ \\
\hline & Partially correct & $7(21)$ & $4(15)$ & \\
\hline Diagnosis of SBP & Correct & $12(35)$ & $21(81)$ & $<0.001$ \\
\hline Correct initiation of treatment & Correct & $12(35)$ & $16(62)$ & 0.040 \\
\hline Urgency of WCC/PMN result & Correct & $13(38)$ & $19(73)$ & 0.007 \\
\hline Knowledge of sources of guidance & Correct & $5(15)$ & $21(81)$ & $<0.001$ \\
\hline
\end{tabular}

Table 2 shows timings related to ascitic fluid samples preand post-intervention. Post-intervention there were a larger number of ascitic fluid samples received at the laboratory (56 pre-intervention compared to 115 post-intervention). The number of individual cirrhotic patients investigated for ascites also increased from 33 pre-intervention to 54 postintervention. The number of taps performed per patient did not differ significantly (median 1.7 compared to 2.1, $\mathrm{p}=0.109$ ). Time between admission and the first diagnostic paracentesis was significantly reduced from a median of $2(0-34)$ days pre-intervention to a median of $1(0-14)$ day post-intervention $(\mathrm{p}<0.001)$. Laboratory time from receiving to completed electronic reporting of samples was also significantly reduced from a median of $3(1-12)$ to $2(0-7)$ days $(\mathrm{p}<0.001)$. There were seven ascitic fluid samples which tested positive for SBP $(\mathrm{PMN}>250$ cells $/ \mathrm{mm}$ or WCC $>500$ cells $/ \mathrm{mm}$ ) in both groups ( 12.5 and $6 \%$, respectively) $(\mathrm{p}=0.151)$. These were from 5 individual patients in the pre-intervention group and 6 in the post-intervention group $(\mathrm{p}=0.461)$.

Following intervention, $63 \%(72 / 115)$ of ascitic samples from cirrhotic patients were labelled to indicate that SBP needed to be excluded. In patients who remained as inpatients for longer than 1 week we also observed an increase in the adherence to weekly repeat paracentesis to monitor for SBP (data not included). The number of cirrhotic patients admitted to the Trust during the 2 six-month periods evaluated did not differ significantly $(213 / 50172$ and $213 / 51525$, respectively $\mathrm{p}=0.823$ ).

Table 2. Summary of findings from review of 6 months of ascitic fluid samples received by the laboratory preand post-intervention.

\begin{tabular}{|c|c|c|c|}
\hline Result & Pre-intervention & Post-intervention & $p$ value \\
\hline Total samples, $n$ & 56 & 115 & - \\
\hline Individual patients, $\mathrm{n}$ & 33 & 54 & - \\
\hline Average number taps/drains per patient & 1.7 & 2.1 & 0.109 \\
\hline Time from admission to tap, median days (range) & $2(0-34)$ & $1(0-16)$ & $<0.001$ \\
\hline Time tap to online reporting, median days (range) & $3(1-12)$ & $2(0-7)$ & $<0.001$ \\
\hline Suspicion of SBP documented on request, n (\%) & $\mathrm{n} / \mathrm{a}$ & $72(63)$ & - \\
\hline Positive samples (PMN > 250 cells/mm) $n(\%)$ & $7(12.5)$ & $7(6)$ & 0.151 \\
\hline Patients diagnosed with SBP, n (\%) & $5(15)$ & $6(11)$ & 0.461 \\
\hline
\end{tabular}




\section{Discussion}

From this review of the AMT trainee doctors' knowledge, we identified a lack of awareness surrounding the suspicion, diagnosis and management of SBP. Given the high mortality associated with inappropriately managed SBP, we felt that intervention to optimise the initial management of cirrhotic inpatients before they are transferred under the management of specialist gastroenterology services was warranted.

To address this lack of awareness, interventions, both educational and clinical, were implemented. A 20-page, stand-alone, educational booklet on SBP was designed using educational concepts developed by Laidlaw, ${ }^{6}$ which emphasise the importance of providing feedback, integration of activity and individualising material to ensure that it is relevant. ${ }^{6,7}$ To ensure that these aims were met we included self-tests to provide feedback to the learner and highlight areas of weakness which they could then focus on individually. A variety of clinical cases were incorporated to ensure that it remained relevant and allowed integration into clinical practice. ${ }^{8} \mathrm{~A}$ senior SpR in gastroenterology then reinforced these concepts by providing a one-hour lecture to the AMT at their weekly teaching session. The session aimed to revisit the basic science surrounding the topic first learned during medical school and integrate this with common clinical scenarios which had presented previously to the Trust. The hope was that this would help trainees incorporate their prior basic science knowledge on SBP with topical, clinically relevant scenarios, thus aiding recall when in future real life clinical situations. ${ }^{9}$ As for clinical interventions, we developed a local diagnostic and management algorithm, based on current EASL and BSG guidance, ${ }^{1,2}$ with accompanying electronic prompts on the investigation requesting system to support trainees in making decisions based on the most up-to-date evidence and enhance their learning for future similar situations.

While we have demonstrated a significant improvement in trainees understanding surrounding SBP in the short term (6 months) several limitations remain in our study. First, we only have a small number of doctors involved in the AMT at our Trust and in July 2014 several were not available to complete the post-intervention questionnaire. However, there was no significant difference in the number of trainees who responded to our questionnaire. Second, given the small event rate of confirmed SBP within the pre- and post-intervention cohorts (5 and 6, respectively), determining any effect on patient outcomes is outside the scope of this study. Finally, we did not formally monitor trainee completion of the educational booklet; therefore we cannot currently evaluate the individual clinical benefit gained from each individual educational and clinical intervention.

Further longitudinal work is now required to determine the long-term outcomes of our interventions on improving the understanding surrounding SBP and clinical outcomes. To achieve this we plan to follow up our current AMT trainees for a further year to assess for maintenance of knowledge postintervention. We also hope to perform further longitudinal work on a larger scale, evaluating the individual impact of individual interventions in the new cohort of trainees who have started regionally from October 2014 to August 2015. This will also provide a large data set of ascitic samples received allowing more detailed analysis of patient outcomes, including the analysis of time to treatment initiation, which firm conclusions could not be drawn on within this study due to the limited sample size.

In conclusion, the current understanding surrounding SBP in trainee doctors involved in the acute management of cirrhotic inpatients in the first 24-72 hours from admission is putting patients at a higher risk of complications and death. Simple interventions to improve awareness, such as targeted education and production of local clinical guidelines and electronic prompts, has significantly improved understanding of SBP in the short term in a cohort of AMT trainee doctors within our Trust.

\section{References}

1 European Association for the Study of the Liver. EASL clinical practical guidelines on the management of ascites, spontaneous bacterial peritonitis, and hepatorenal syndrome in cirrhosis. $J$ Hepatol 2010;53:397-417.

2 Moore KP, Aithal GP. Guidelines on the management of ascites in cirrhosis. Gut 2006;55;1-12.

3 Kim JJ, Tsukamoto MM, Mathur AK et al. Delayed paracentesis is associated with increased in-hospital mortality in patients with spontaneous bacterial peritonitis. Am J Gastroenterol 2014;109:1436-42.

4 Bhala N, Aithal G, Ferguson J. How to tackle rising rates of liver disease in the UK. BMJ 2013;8;f807.

5 Volk ML, Tocco RS, Bazick J, Rakoski MO, Lok AS. Hospital readmissions among patients with decompensated cirrhosis. Am J Gastroenterol 2012;107:247-52.

6 Laidlaw JM. Principles of learning IMD2. Dundee: Centre for Medical Education, 1995.

7 Hartley S, Gill D, Carter F, Walters K, Bryant P. Teaching medical students in primary and secondary care. Oxford: Oxford University Press, 2003.

8 Laidlaw JM and Harden RM. What is...a study guide? Med Teach 1990;12:7-12.

9 Schmidt HG. Foundations of problem-based learning: some explanatory notes. Med Educ 1993;27:422-32.

Address for correspondence: Dr TM Rawson, Commonwealth Building, Hammersmith Hospital, Du-Caine Road, London W12 0NN, UK. Email: tmr07@ic.ac.uk 